\title{
Bei wem und warum nach Proteinurie fahnden?
}

\author{
Die Proteinurie signalisiert nicht nur eine renale Schädigung, sondern ist auch ein Risiko- \\ marker für kardiovaskuläre Ereignisse und somit von prognostischer Relevanz. Bei welchen \\ Patienten ist es sinnvoll danach zu fahnden, und welche Konsequenzen ergeben sich aus \\ dem Nachweis einer Proteinurie?
}

Eine Proteinurie entsteht meist durch einen undichten Filter, ist also Folge einer glomerulären Schädigung“, sagte Prof. Michael Mayr, Basel. Aber auch ein tubulärer Schaden kann zu einer Proteinurie führen, weil sezernierte Proteine teilweise tubulär rückresorbiert werden. Eine Proteinurie von 40-80 mg/Tag, davon ca. $10 \mathrm{mg}$ Albumin, ist physiologisch. Bei einer Proteinurie bis $300 \mathrm{mg} /$ Tag spricht man von einer Mikroalbuminurie oder "moderate proteinuria“, bei einem Wert darüber von einer Makroalbuminurie oder „overt proteinuria“ und ab einer Ausscheidung von 3-3,5 g/ Tag von einer Proteinurie im nephrotischen Bereich.

Qualitativ unterscheidet man die glomeruläre Proteinurie, die vorwiegend aus Albumin besteht, und die tubuläre Proteinurie mit Ausscheidung von alpha-1-Mikroglobulin, z. B. bei einer interstitiellen Nephritis, sowie die Paraproteinurie. „Diese Formen können auch kombiniert vorkommen“, erklärte Mayr. Überdies muss man differenzialdiagnostisch auch die post- und extrarenale Proteinurie, z. B. bei hämorrhagischer Zystitis, Menstruation, Nephrolithiasis oder Tumoren, berücksichtigen.

Die häufigsten Ursachen einer Proteinurie sind Diabetes und Hypertonie, seltener sind Glomerulonephritiden und Vaskulitiden. „Differenzialdiagnostisch sollte auch immer eine orthostatische Proteinurie diskutiert und ausgeschlossen werden“, so Mayr.

\section{Vor- und Nachteil des Streifentests}

Standard zum Proteinurienachweis ist der Streifentest, der aber nur Albumin erfasst und somit eine gute Spezifität, aber eine geringe Sensitivität aufweist. Er wird erst ab einer Albuminausscheidung von ca. 200-300 mg/l positiv, d.h. die Mikroalbuminurie wird damit nicht erfasst. „Das Problem des Streifentests ist die ungenaue Quantifizierung der Proteinurie, da die gemessene Konzentration volumenabhängig ist", so Mayr. Goldstandard ist die Bestimmung der Proteinausscheidung im 24-Stunden-Urin, die jedoch mühsam und fehleranfällig ist. Praktikabler ist die Bestimmung des Protein/ Kreatinin- bzw. des Albumin/KreatininQuotienten im Spot-Urin.

\section{Screening-Kandidaten}

Die Bestimmung der Eiweißausscheidung im Urin erfolgt entweder als Screening, zur Diagnosefindung bei symptomatischen Patienten oder zum Monitoring i.S. einer Verlaufsbeurteilung. Ein Screening sollte nur gezielt bei $>60$-Jährigen, bei Hypertonikern und Diabetikern durchgeführt werden, um das kardiovaskuläre und renale Risiko früh $\mathrm{zu}$ erfassen. Bei Nachweis einer Proteinurie sollte eine Therapie mit einem ACEHemmer- oder $\mathrm{AT}_{1}$-Blocker begonnen werden. „Bei Typ-1-Diabetikern sollte fünf Jahre nach Diagnosestellung, bei Typ-2-Diabetikern bereits bei der Diagnosestellung das erste Mal ein Screening durchgeführt werden“, so Mayr. Bei einem negativen Befund empfehle sich eine Kontrolle in jährlichen Abständen.

Wird der Patient schon mit einem RAS-Blocker behandelt, sei ein Screening nicht nötig. Der Sinn des Screenings ergebe sich allein daraus, durch frühzeitige Gabe eines RAS-Blockers den Verlauf der diabetischen Nephropathie günstig zu beeinflussen. Die Kombination eines ACE-Hemmers mit einem $\mathrm{AT}_{1^{-}}$ Blocker wird nicht mehr empfohlen. Als Zielblutdruck gilt heute auch bei Diabetikern ein Wert < 130/80 mm Hg.

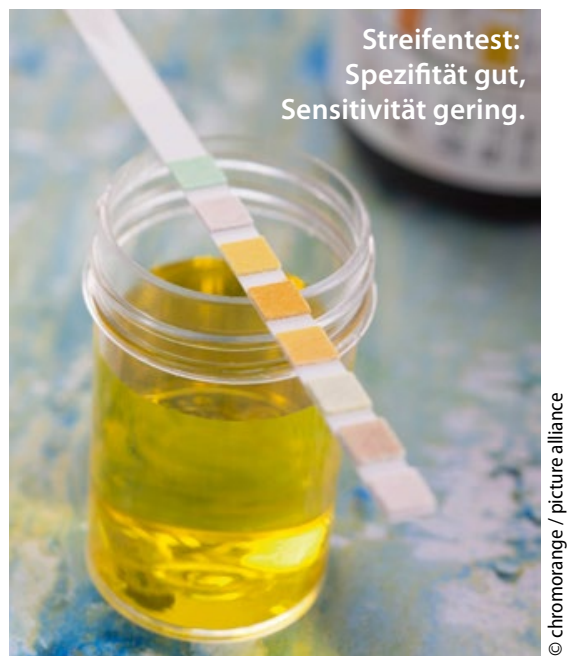

Bei Patienten mit chronischer Niereninsuffizienz, glomerulärer Hämaturie oder morphologischen Pathologien gehört die Bestimmung der Proteinurie zum unverzichtbaren diagnostischen $\mathrm{Ba}$ sisprogramm, um die Erkrankung ätiologisch und somit auch therapeutisch und prognostisch richtig zuordnen $\mathrm{zu}$ können. Die Bestimmung des Albumin/ Kreatinin-Quotienten eignet sich zudem zur Beurteilung des Therapieerfolgs, sodass bei entsprechenden Erkrankungen auch ein Monitoring sinnvoll ist.

Dr. Peter Stiefelhagen

- Quelle: medArt, 23.6.2015 in Basel

Mehr zum Thema auf SPRINGERMEDIZIN.DE

Schwerpunkt Nephrologie Niereninsuffizienz, Hypertonie, Elektrolyte, Transplantation: In dieser ständig aktualisierten Themensammlung dreht sich alles um die Niere:

$\rightarrow$ www.springermedizin.de/299046 